\title{
Retrospective cohort study comparing current third stage management to expedient squatting third stage management
}

\author{
Judy Cohain ${ }^{1}$ and Rina E. Buxbaum ${ }^{2}$ \\ ${ }^{1}$ Hebrew University \\ ${ }^{2}$ Hadassah Medical Center
}

February 16, 2021

\begin{abstract}
Abstract Objective: To compare current the third stage management to expedient squatting at 3 minutes postpartum. Design, Setting, Sample and Methods: A retrospective cohort study of 1,098 planned, attended low risk vaginal births in Israel using Judy's 3,4,5 minute third stage protocol compared to 2,691 attended low risk vaginal births in British Columbia using various forms of active or expectant management of the third stage of labor. Main Outcome measures: PPH>1000, PPH $>500$ and manual removal of placenta Results: Among similar groups of low risk births, active management, or expectant management resulted in 4.1\% PPH over 1000 cc, whereas Judy's 3,4,5 minute protocol resulted in 0\% PPH over 500 cc. Conclusion: Evidence supports less postpartum bleeding and postpartum hemorrhage when women deliver the placenta in squatting 3 minutes after birth. The risks are minimal and the data suggests the likelihood of a very positive outcome, making it recommended for practitioners in all settings to try it.
\end{abstract}

Retrospective cohort study comparing current third stage management to expedient squatting third stage management

Authors: Judy Slome Cohain, CNM

Address: judyslome@hotmail.com

Affiliation: Private Practice- corresponding author

Snail mail: Alon Shvut 37, Israel 90433 Telephone 972545493109

Second author: Rina E. Buxbaum, MD rina1511@gmail.com

Affiliation: Hadassah Ein Kerem Medical Center

A shortened running title : Preventing PPH using expedient squatting

Abstract

Objective: To compare current the third stage management to expedient squatting at 3 minutes postpartum.

Design, Setting, Sample and Methods: A retrospective cohort study of 1,098 planned, attended low risk vaginal births in Israel using Judy's 3,4,5 minute third stage protocol compared to 2,691 attended low risk vaginal births in British Columbia using various forms of active or expectant management of the third stage of labor.

Main Outcome measures: $\mathrm{PPH}>1000, \mathrm{PPH}>500$ and manual removal of placenta

Results: Among similar groups of low risk births, active management, or expectant management resulted in 4.1\% PPH over $1000 \mathrm{cc}$, whereas Judy's 3,4,5 minute protocol resulted in 0\% PPH over $500 \mathrm{cc}$. 
Conclusion: Evidence supports less postpartum bleeding and postpartum hemorrhage when women deliver the placenta in squatting 3 minutes after birth. The risks are minimal and the data suggests the likelihood of a very positive outcome, making it recommended for practitioners in all settings to try it.

* Introduction

Cochrane reviews found no evidence that active management reduces the risk of primary postpartum hemorrhage (PPH) over $1000 \mathrm{cc}$ and found active management increases afterbirth pains, increases the need for postpartum analgesia, increases the incidence of postpartum diastolic blood pressure greater than $90 \mathrm{mmHg}$, and results in increased rehospitalizations due to bleeding. [1] The PPH rate at vaginal birth was $5 \%$ both before and after the adoption of Active Management. [2-4] Active Management appears to have superior outcomes only when compared to the $13 \% \mathrm{PPH}$ rates associated with Expectant Management. [1] The failure of Active Management to prevent PPH is not surprising since it is based on the presumption that women evolved to require external administration of synthetic Oxytocin to avoid hemorrhage. Humans share the same placental physiology as apes and chimpanzees who never hemorrhage unless the birth is interfered with by humans. $[5,6]$ It follows that if humans deliver the placenta the way apes and chimps do, they also would not hemorrhage. A demonstration of this appears on YouTube: https://www.youtube.com/watch?v=AAJPW4p6rzU

see $* * * *$

The objective of this retrospective cohort study was to compare the efficacy of Judy's 3,4,5 minute third stage algorithm to variations of active and expectant third stage management among similar homebirth populations during the same time period.

* Methods Two populations with identical participant selection criteria, similar labor management, and different third stage management were compared. Both groups consist of women planning attended homebirths in countries with free, socialized medicine, with midwives who are similarly trained to limit medical interventions in labor. PPH was defined as blood loss over $1000 \mathrm{cc}$ in the first 2 hours after birth in both groups. In the study group, estimated blood loss was documented to the nearest $5 \mathrm{cc}$ for all births.

The study group was all births attended from Jan. 1, 2000- Dec. 31, 2020 by the All The Way Home Birth Service serving the Jewish population in Israel, a population of approximately 5 million. All midwives working for All The Way Home Birth Service are formally trained and licensed. All The Way Home birth practice provides free homebirth to any qualified woman who cannot afford the generally affordable fee. Each birth outcome was recorded in a patient record and an Excel spreadsheet immediately following the birth. The criteria for inclusion as a patient in both homebirth practices were: Singleton fetus; cephalic presentation; gestational age greater than 36 and less than 41 completed weeks of pregnancy; spontaneous onset of labor; history of up to one previous cesarean; absence of significant pre-existing disease including heart disease, hypertensive chronic renal disease or type 1 diabetes; absence of significant disease arising during pregnancy including hypertension disorders during pregnancy with proteinuria $(>0.3 \mathrm{~g} / \mathrm{L}$ by urine dipstick), antepartum hemorrhage after 20 weeks' gestation, gestational diabetes requiring insulin, active genital herpes, placenta previa or placental abruption. All participants gave written informed consent to deliver the placenta using Judy's 3,4,5 minute algorithm and to have their outcomes anonymously published. The study group's third stage was managed by cutting the cord exactly 3 minutes after the birth if the woman consents to it, pushing out the placenta in squatting at 3 minutes; getting into bed by 4 minutes and checking the abdomen to make sure the uterus is contracted at 5 minutes and to massage it to contract if it is not. A prophylactic $0.125 \mathrm{mg}$ pill of Methergine was given orally at 10 minutes postpartum to women who had lost $250 \mathrm{cc}$ by 10 minutes postpartum.

The first 500 births were delivered on plastic sheets without absorbent pads, measuring blood loss with a measuring cup. Clots of blood were collected, formed into a ball and blood loss calculated according to the diameter of the ball: $3 \mathrm{~cm}$ diameter round clot $=15 \mathrm{cc}$ blood loss, $4 \mathrm{~cm}=33 \mathrm{cc} ; 5 \mathrm{~cm}=65 \mathrm{cc}$ blood loss; $6 \mathrm{~cm}=113 \mathrm{cc}, 7 \mathrm{~cm}=180 \mathrm{cc} ; 8 \mathrm{~cm}$ diameter ball $=270 \mathrm{cc}$ blood loss; $9 \mathrm{~cm}=380 \mathrm{cc} ; 10 \mathrm{~cm}=523 \mathrm{cc} ; 12$ $\mathrm{cm}=900 \mathrm{cc}$ blood loss. Both PPH over $500 \mathrm{cc}$ and over 1000cc were documented. 
Janssen et. al. 2009 was selected as the control group because it reports the lowest PPH rate among the high-quality homebirth studies. [17,18] Janssen et.al. 2009 most closely matches the study group in timeperiod, Jan. 1, 2000, to Dec. 31, 2004, population, and birth management and most importantly, had identical inclusion criteria to the study group. British Columbia, a province of Canada, had a population of 4.4 million at the time of the study. Like Israel, midwifery care is accessible to all women in the province who meet the standards for low obstetric risk. The midwives in British Columbia use variations and combinations of active and expectant management. [19] Their database defines PPH as [?]1000 mL blood loss. [20]

The primary outcome measure was the rate of postpartum hemorrhage over $1000 \mathrm{cc}$. Secondary outcome measure was manual removal of placenta. Formal ethical approval of this research was received from the Hadassah Medical Organization Helsinki Committee Institutional Review Board on April 14, 2014.

* Results

During the study period, 2,899 women attended by a registered midwife began labour with the intention of giving birth at home in British Columbia and 2,691 did so, using currently accepted third stage management; 1,098 women attended by a registered midwife began labor with the intention of giving birth at home in Israel of which 1,093 births did so using the 3,4,5 third stage protocol. Patient demographics- see Table 1.

Variations of active and expectant third stage protocols resulted in $4 \%(110 / 2899) \mathrm{PPH}$ over $1000 \mathrm{cc}$ in the first two hours. The 345 protocol resulted in zero PPH $>1000 \mathrm{cc}$ in the first two hours, i.e. active/expectant involves a 20 times higher risk of PPH compared to expedient squatting.

The 3,4,5 minute third stage protocol resulted in blood losses of an average of $100 \mathrm{cc}$ in the first hour; one $\mathrm{PPH}=800 \mathrm{cc}$ in the first hour of a grandmultiparous woman with no anemia or history of hemorrhages, but whose birth followed shortly after the violent murder of her brother. After the birth of the placenta, she screamed uncontrollably and continuously for 20 minutes that she wished she was dead. This made it appear that PPH was psychological in origin. The other PPH was $500 \mathrm{cc}$, a late PPH starting 5 hours after birth in a 26 year old, P3, 41+1, after a 16 hour labor, $3600 \mathrm{gm}$ baby, with hemoglobin level of $8.0 \mathrm{~g} / \mathrm{L}$ at the start of labor. She received one unit of blood. No negative side effects of third stage squatting protocol were observed among 1,093 vaginal births.

Discussion

Main findings:

In the study population, Judy's 3,4,5 third stage protocol decreased average postpartum bleeding in the first hour from $500 \mathrm{cc}$ to $100 \mathrm{cc}$; decreased PPH over $1000 \mathrm{cc}$ from $4 \%$ to $0 \%$; and did so without negative side effects or financial expense. The 345 is logical, has been tried on a large sample, and has been proven to reduce PPH as well as average blood loss. The habit of laying supine to deliver the placenta results in at least $4 \% \mathrm{PPH}$ of over a liter of blood. No mother objected to not holding her baby for the one minute that it takes to deliver the placenta in squatting. Several fathers were raving after the birth about how special it was for them to hold a baby so soon after birth. As practitioners garnered more experience with expedient squatting, less oral methergine was administered.

No other protocol has reported an average blood loss of less than $500 \mathrm{cc}$ in the first hour and $0 \%$ postpartum hemorrhage over $1000 \mathrm{cc}$. [21] Without exception, all other low risk populations report higher PPH rates: $4.4 \% \mathrm{PPH}$ over 1000 cc. [19] 4.6\% PPH over 1000 cc. [22] 9.3\% over 500 cc. [23] and $11.7 \% \mathrm{PPH}$ over 500 cc. [24] The two largest high quality homebirth studies: Birthplace and DeJonge et.al. did not document PPH rates at all. $[25,26]$ Large hospital studies looking at multiple sites and reporting on tens of thousands of vaginal births, continue to report $5 \% \mathrm{PPH}$ at vaginal birth. [4]

Strengths and limitations:

In the study group, $20 \%$ of labors were augmented with bilateral nipple massage which results in oxytocin receptor stimulation, the goal of IV Pitocin. The lack of use of IV Pitocin in the study group to augment labor is unlikely to explain a $0 \%$ PPH because prior to the synthesis and use of Pitocin starting in 1953, the 
$\mathrm{PPH}$ rate was also 5\% [2] and PPH rates in the absence of Pitocin augmentation in hospital are currently 4\%. [4] Theories extrapolated from in vitro studies suggest that Pitocin augmentation in labor causes PPH because it attenuates oxytocin-induced contractility of human myometrium. [27-29] A theory for how Pitocin continuously augments uterine contractions in labor, yet immediately after birth the receptors that were sensitive to Pitocin become insensitive to Pitocin, is lacking. The association of Pitocin augmentation with PPH might be due to an increased delay in delivery of the placenta at births augmented with Pitocin, perhaps due to a feeling that the freely flowing IV Pitocin will protect the woman from PPH.

The published association between longer duration of the second stage as causing increased PPH also lacks a logical theory because indeed, during long second stages, the uterus continues effectively contracting until the fetus emerges and no reason has been provided for why it should not contract afterwards. [30] Long second stages are usually a result of a large fetus or an anesthetized or unmotivated woman. Where women push for 15, 17 and even 22 hours, no increase in PPH was observed as long as the placenta is delivered within 5 minutes of the birth. [31]

A critique of squatting protocol, by practitioners who have never tried the 345 , is that the study group midwives are underreporting excess postpartum bleeding and PPH. Underreporting of PPH is suspected even in the highest quality studies. [1] The best definition of PPH is a larger than $20 \mathrm{~g} / \mathrm{L}$ drop in Hgb level compared to the level before the birth but this requires documentation of routine and reliable blood testing before and after the birth. Erickson et.al. rightfully suggests the term PPH applies to both PPH over $500 \mathrm{cc}$ and those over $1000 \mathrm{cc}$ because it is challenging or impossible to distinguish between them [page 610] since both $600 \mathrm{cc}$ and $1050 \mathrm{cc}$ of blood cover an entire 60X90 pad. However, a $100 \mathrm{cc}$ blood loss does not cover any entire pad. It is therefore impossible to confuse $100 \mathrm{cc}$ blood loss with blood loss of $500 \mathrm{cc}$ at birth. Blood loss of $100 \mathrm{cc}$ in the first hour typically means one tablespoon attached to the placenta, another $35 \mathrm{cc}$ on the 60x90 pad she gives birth on, and 4 tablespoons or $50 \mathrm{cc}$ seen one hour after birth on the pad used starting from 5 minutes after birth when she got into bed. The knee jerk excuse to discard an overlooked protocol cannot be applied to this protocol.

The study group had fewer nulliparas: $26 \%$ vs. $44 \%$. Nulliparity is associated with higher rates of PPH. [4] The difference in nulliparity rates could account for a slightly lower PPH rate in the study group but not the dramatic reduction found. On the other hand, although the control group did not report grandmultiparity, it is likely the study group had a higher rate of grandmultiparity, as the fertility rate in Israel is higher than in British Columbia. Grandmultiparity is associated with higher rates of PPH. [4] No increase in PPH was found among grandmultiparas when Judy's 3,4,5 was used. The findings suggest the associations with PPH pinned on nulliparity or grandmultiparity are due to other qualities of nulliparous and grandmultiparous births since they disappear when the placenta is delivered at 3 minutes.

\section{Interpretation}

Except for exceptional cases, women appear not to experience $\mathrm{PPH}>500 \mathrm{cc}$ where the placenta is delivered in squatting expediently.

Conclusion:

Evidence supports less postpartum bleeding and postpartum hemorrhage when women deliver the placenta in squatting 3 minutes after birth. The risks are minimal and the data suggests the likelihood of a very positive outcome, making it recommended for practitioners in all settings to try it.

Acknowledgements:

Ethics approval and consent to participate- Formal ethical approval of this research was received from the Hadassah Medical Organization Helsinki Committee Institutional Review Board on April 14, 2014. All methods were performed in accordance with guidelines and regulations provided in the ethics approval. All participants gave written consent to participate and have their outcomes published anonymously. Consent for publication- all participants gave written consent for their outcomes to be published anonymously. 
Availability of data and materials- The datasets used and analyzed during the current study available from the corresponding author on reasonable request. Competing interests- all authors declare no competing interests.

Funding- all expenses out of pocket, No funding was provided by anyone else.

Authors' contributions- JSC wrote most of the paper. REB did analysis and proofing the paper.

Acknowledgments- thanks to all participants, authors, peer reviewers and editor.

References

1. Begley CM, Gyte GML, Devane D et.al. Active versus expectant management for women in the third stage of labour. Cochrane Database of Systematic Reviews 2019,Issue 2. Art. No.: CD007412

2. Melody GF. Primary postpartum hemorrhage. Calif Med. 1951;75(6):425-429.

3. Magann EF, Evans S,Chauhan SP et.al.The length of the third stage of labor and the risk of postpartum hemorrhage.Obstet Gynecol. 2005;105(2):290-3.

4. Erickson EN, Lee CS, Carlson NS. Predicting Postpartum Hemorrhage After Vaginal Birth by Labor Phenotype. J Midwifery Womens Health. 2020;65(5):609-620.

5. Carter AM, Pijnenborg R. Evolution of invasive placentation with special reference to non-human primates. Best Pract Res Clin Obstet Gynaecol. 2011;25(3):249-57.

6. Abrams ET, Rutherford JN. Framing postpartum hemorrhage as a consequence of human placental biology: an evolutionary and comparative perspective. Am Anthropol. 2011. 113, 3, 417-30.

7. Chamberlain G. British maternal mortality in the 19th and early 20th centuries. J R Soc Med. 2006;99(11):559-563.

8. Abrams ET, Rutherford JN. Framing postpartum hemorrhage as a consequence of human placental biology: an evolutionary and comparative perspective. Am Anthropol. 2011. 113, 3, 417-30.

9. https://medicalxpress.com/news/2020-10-genetic-postpartum-hemorrhage.html Last accessed Feb 4,2021.

10. Anderson JM, Etches D. Prevention and management of postpartum hemorrhage. Am Fam Physician. 2007;75(6):875-82.

11. Neiman E, Austin E, Tan A et.al. Outcomes of Waterbirth in a US Hospital-Based Midwifery Practice: A Retrospective Cohort Study of Water Immersion During Labor and Birth. J Midwifery Womens Health. 2020;65(2):216-223.

12. Williams JW, Pritchard JA, MacDonald PC (1980). Williams Obstetrics. 16th ed. New York:AppletonCentury-Crofts.

13. Cohain JS. A Proposed Protocol for Third Stage Management-Judy's 3,4,5,10 minute method. Birth 2010; 37(1)84-5.

14. Cohain JS. Back to Basics to Eliminate Postpartum Hemorrhage at Vaginal Birth. Birth 2016; 43(1)93.

15. Cohain JS. Minimizing bleeding and tearing at vaginal births. Women and Birth 2018;31(2):e144.

16. Cohain JS. Expedient squatting third-stage technique to prevent excessive bleeding at birth. MIDIRS Midwifery Digest. 2020;30(4):495-6

17. Cohain JS. Instead of attacking homebirth, hospital practitioners should be trying to understand why their outcomes compare unfavorably to homebirth outcomes. BJOG. 2016;123(7):1231.

18. Janssen PA, Saxell L, Page LA et.al. Outcomes of planned home birth with registered midwife versus planned hospital birth with midwife or physician. CMAJ 2009;181(6-7):377-383. 
19. Tan WM, Klein MC, Saxell L, Shirkoohy SE, Asrat G. How do physicians and midwives manage the third stage of labor? Birth. 2008;35(3):220-9.

20. Janssen PA, Lee SK, Ryan EM et.al. Outcomes of planned home births versus planned hospital births after regulation of midwifery in British Columbia. CMAJ. 2002;166(3):315-323.

21. Fahy KM. Third stage of labour care for women at low risk of postpartum haemorrhage. J Midwifery Womens Health 2009;54(5): 380-386.

22. Bolten N, de Jonge A, Zwagerman E et.al. Effect of planned place of birth on obstetric interventions and maternal outcomes among low-risk women: a cohort study in the Netherlands. BMC Pregnancy Childbirth. 2016;16(1):329.

23. Hutton EK, Reitsma AH, Kaufman K. Outcomes associated with planned home and planned hospital births in low-risk women attended by midwives in Ontario, Canada, 2003-2006: a retrospective cohort study. Birth. 2009;36(3):180-189.

24. Campiotti M, Campi R, Zanetti M et.al. Low-Risk Planned Out-of-Hospital Births: Characteristics and Perinatal Outcomes in Different Italian Birth Settings. Int J Environ Res Public Health. 2020;17(8):2718.

25. Birthplace in England Collaborative Group, Brocklehurst P, Hardy P et.al. Perinatal and maternal outcomes by planned place of birth for healthy women with low risk pregnancies: the Birthplace in England national prospective cohort study. BMJ. 2011;343:d7400.

26. de Jonge A, Peters L, Geerts CC et.al. Mode of birth and medical interventions among women at low risk of complications: A cross-national comparison of birth settings in England and the Netherlands. PLoS One. 2017;12(7):e0180846.

27. Balki M, Ramachandran N, Lee S, Talati C. The recovery time of myometrial responsiveness after oxytocin-induced desensitization in human myometrium in vitro. Anesth Analg. 2016;122(5):1508-1515.

28. Balki M, Erik-Soussi M, Kingdom J, Carvalho J. Oxytocin pretreatment attenuates oxytocin-induced contractions in human myometrium. Anesthesiology. 2013;119(3):552-561.

29. Talati C, Carvalho JCA, Luca A, Balki M. The effect of intermittent oxytocin pretreatment on oxytocininduced contractility of human myometrium in vitro. Anesth Analg. 2019;128(4):671-678.

30. Dionne MD, Deneux-Tharaux C, Dupont C, Duration of expulsive efforts and risk of postpartum hemorrhage in nulliparous women: a population-based study. PLoS One. 2015;10(11):e0142171.

31. Cohain JS. Three cases of Prolonged Second Stage of Labor 14, 17 and 24 hours. Midwifery Today. 2017:122:18-20. ****Additionals to background:

The following further additional comments in background are only to answer the inquiries of previous peer review:

The protocol to prevent all PPH over $1000 \mathrm{cc}$ at vaginal birth has been in the medical literature since 2010. The most common objection to the theory that all PPH is preventable is the misconception that in previous centuries women died at birth in droves from PPH, not infection. The evidence shows that infection caused the deaths of 1.5\% of mothers 1911-1935 in the United Kingdom (UK). Maternal death from PPH in the UK in that period was the very rare result of malpractice such as a poorly managed low lying placenta or trauma to the cervix or uterus at delivery. [7] Only in the past few decades is postpartum hemorrhage the reason for the majority of maternal deaths, with rates of PPH increasing year by year in the past decade, mostly from the overuse of cesarean surgery. There continues to be a desire to find a genetic basis of PPH. [8,9] It would be more reasonable to assume that any genes involved in primate PPH were eliminated by natural selection millions of years ago since PPH is a deadly trait.

Birth is the only circumstance for which losing $500 \mathrm{cc}$ of blood is considered acceptable. The Cochrane review explains that the current average blood loss of $500 \mathrm{cc}$ at vaginal birth is acceptable since people who 
donate blood also lose $500 \mathrm{cc}$ without deleterious effects. [1] This is a strange justification in light of the facts that blood donation is not done to improve health but rather a compromise of one person's well-being to improve anothers; pregnant women are ineligible to donate blood; postpartum blood loss is not an exact amount like blood donation; and postpartum blood losses of $500 \mathrm{cc}$ in the first hour can be mistaken for actual blood loss of $1000 \mathrm{cc}$. [1,4] It would be more logical to define and treat postpartum blood loss the way all other types of bleeding: as deleterious and worthy of preventing.

There is a direct relationship between PPH $>1000 \mathrm{cc}$ and the length of the third stage such that PPH increases linearly with the passing of every second of third stage [3,10] Studies showing this linear relationship failed to conclude that shortening the third stage would prevent PPH. [3,11] Except in the rare case of placenta accreta, the placenta detaches within a minute of the birth as a result of the dramatic change in shape of the uterus as the baby delivers. [12] In current practice, women lay supine in bed with the heavy, flaccid, detached placenta lying on the posterior or dorsal wall of the uterus below the level of the cervix while the wound where the placenta was previously attached, is bleeding into the uterus and the cervix is closing. When the woman is supine, the detached placenta has an uphill ascent against the forces of gravity. Requiring the placenta to ascend against gravity sometimes delays the delivery. Any delay provides additional time for the wound to bleed as well as additional time for the cervix to close. A closing cervix creates the need to squeeze the placenta through a narrower passage, sometimes leaving bits behind resulting in PPH due to retained placenta. Delivering the placenta at 3 minutes after birth in squatting, uses expedience, gravity, the downward force of the diaphragm and the abdominal muscles to deliver the placenta completely and before uterine atony or placental retention can cause excess bleeding. [13-16] The woman gets into squatting at 3 minutes after the birth and the practitioner reminds her to push out the placenta without waiting for the sensation of a uterine contraction. There is no reason not to cut the cord unless the newborn is not breathing and requires resuscitation. By 1 minute after birth, the placenta has detached from the uterus and newborn cord/placental circulation has been shunted to the lungs. However, where there is objection to cutting the cord at 3 minutes, the woman can squat and deliver the placenta with the cord attached to the newborn. Once the complete placenta is delivered, the uterus contracts thereby closing off uterine blood vessels that previously fed the placenta, preventing uterine atony, the cause of the majority of $\mathrm{PPH}$. The protocol can be carried out in any setting, requiring no equipment other than a way to accurately time 3 minutes, such as a digital watch. It is likely that less bleeding would occur if the woman squatted BEFORE 3 minutes but in general, women appreciate three minutes to recover from the birth of the baby.

1

\section{Hosted file}

patient demographics.xlsx available at https://authorea.com/users/305391/articles/509301retrospective-cohort-study-comparing-current-third-stage-management-to-expedientsquatting-third-stage-management 